\title{
Numerical analysis of a non-singular boundary integral method: Part I. The circular case
}

\author{
P. Dreyfuss* and J. Rappaz \\ Département de Mathématiques, EPFL, 1015 Lausanne, Switzerland
}

Communicated by J. C. Nedelec

\begin{abstract}
SUMMARY
In order to numerically solve the interior and the exterior Dirichlet problems for the Laplacian operator, we present here a method which consists in inverting, on a finite element space, a non-singular integral operator. This operator is a geometrical perturbation of the Steklov operator, and we precisely define the relation between the geometrical perturbation and the dimension of the finite element space, in order to obtain a stable and convergent scheme. Furthermore, this numerical scheme does not give rise to any singular integral.

The scheme can also be considered as a special quadrature formula method for the standard piecewise linear Galerkin approximation of the weakly singular single layer potential, the special quadrature formula being defined by the introduction of a neighbouring curve.

In the present paper, we prove stability and we give error estimates of our numerical scheme when the Laplace problem is set on a disk. We will extend our results to any domains by using compact perturbation arguments, in a second paper. Copyright (C) 2001 John Wiley \& Sons, Ltd.
\end{abstract}

\section{INTRODUCTION}

Let $\Omega$ be a simply connected, bounded, open domain in $\mathbb{R}^{2}$, the boundary $\Gamma$ of which is assumed regular. For the sake of simplicity, we will suppose in the following that $\Gamma$ is $\mathscr{C}^{\infty}$. The spaces $H^{1}(\Omega), H^{1 / 2}(\Gamma), H^{-1 / 2}(\Gamma)$ will denote the classical Sobolev spaces of functions on $\Omega$ or $\Gamma$. The duality $H^{-1 / 2}(\Gamma), H^{1 / 2}(\Gamma)$ will be denoted by $\langle., .\rangle_{-1 / 2,1 / 2}$. If $u_{0}$ is given in $H^{1 / 2}(\Gamma)$, we are looking for a function $u$ in $H^{1}(\Omega)$ satisfying

$$
\begin{array}{rr}
\Delta u=0 & \text { in } \Omega \\
u=u_{0} & \text { on } \Gamma
\end{array}
$$

\footnotetext{
*Correspondence to: P. Dreyfuss, Département de Mathématiques, EPFL, 1015 Lausanne, Switzerland

Contract/grant sponsor: Swiss National Fund for Scientific Research
}

Copyright (c) 2001 John Wiley \& Sons, Ltd.

Received 1 February 2001 
It is well known that problem (1) has a unique solution $u$ and it is easy to show that if $x$ does not belong to $\bar{\Omega}=\Omega \cup \Gamma$, then we have

$$
\forall x \notin \bar{\Omega}: \int_{\Gamma} G(x, y) \frac{\partial u}{\partial n_{y}}(y) \mathrm{d} s_{y}=\int_{\Gamma} \frac{\partial G}{\partial n_{y}}(x, y) u_{0}(y) \mathrm{d} s_{y}
$$

where $G$ denotes the Green kernel and $\partial G / \partial n_{y}$ its external normal derivative with respect to the variable $y$; i.e. if $x \in \mathbb{R}^{2}, y \in \Gamma$ :

$$
\begin{aligned}
G(x, y) & =-\frac{1}{2 \pi} \log |x-y| \\
\frac{\partial G}{\partial n_{y}}(x, y) & =-\frac{\left(x-y, n_{y}\right)}{2 \pi|x-y|^{2}}
\end{aligned}
$$

where $(.,$.$) is the scalar product, |$.$| the Euclidian norm in \mathbb{R}^{2}$ and $n_{y}$ the outward unit normal vector at point $y$.

Let us assume that we are only interested in knowing the normal derivative $\partial u / \partial n_{y}$ on $\Gamma$ instead of the whole $u$ in $\Omega$. Then if $\tilde{\Gamma}$ is the boundary of a regular, simply connected, bounded, open domain $\tilde{\Omega}$ containing $\bar{\Omega}$, and if we call $\zeta$ the unknown $\partial u / \partial n_{y}$ on $\Gamma$, the integral of which is vanishing, we are in the position to give a variational formulation of (2).

Find $\zeta \in H_{0}^{-1 / 2}(\Gamma)$ satisfying

$$
\forall \mu \in H_{0}^{-1 / 2}(\tilde{\Gamma}): \int_{\tilde{\Gamma}} \mathrm{d} s_{x} \int_{\Gamma} \mathrm{d} s_{y} G(x, y) \zeta(y) \mu(x)=\int_{\tilde{\Gamma}} \mathrm{d} s_{x} \int_{\Gamma} \mathrm{d} s_{y} \frac{\partial G}{\partial n_{y}}(x, y) u_{0}(y) \mu(x)
$$

where $H_{0}^{-1 / 2}(\Gamma)=\left\{\gamma \in H^{-1 / 2}(\Gamma):\langle\gamma, 1\rangle_{-1 / 2,1 / 2}=0\right\}$. Clearly speaking, problem (5) posseses at least one solution $\zeta$ because it suffices to take $\zeta=\partial u / \partial n_{y}$ when $u$ is the solution of problem (1). The uniqueness is a delicate thing (see Reference [1] for instance).

Our numerical method is built on the Galerkin approximation of integral formulation (5) called 'Kupradze integral equation'. Historically, this formulation was introduced by Kupradze (cf. Reference [2]). Since this equation is ill-posed, classical numerical methods to solve it are very unstable. This fact was shown by Christiansen (cf. References [3,4]). The Tikhonov regularization can be used in order to put right this situation but we obtain a method which converges slowly (cf. Reference [5, p.327]). Nevertheless, engineers have empirically found how to construct stable and effective numerical methods (cf. Reference [6]), which are sometimes used nowadays. In order to explain it, we define the Hilbert spaces $X$ and $Y$ by $X=H_{0}^{-1 / 2}(\Gamma)$ and $Y=H_{0}^{-1 / 2}(\tilde{\Gamma})$. In view of numerically solving problem (5), we define the continuous bilinear form $\tilde{a}: X \times Y \rightarrow \mathbb{R}$ by

$$
\zeta \in X, \mu \in Y: \tilde{a}(\zeta, \mu)=\int_{\tilde{\Gamma}} \mathrm{d} s_{x} \int_{\Gamma} \mathrm{d} s_{y} G(x, y) \zeta(y) \mu(x)
$$

Unfortunately, the bilinear form $\tilde{a}(.,$.$) does not satisfy the 'inf-sup' conditions of Babuska-$ Ladyzenskaja (see Reference [7] for instance) because the problem of finding $\zeta \in X$ satisfying $\tilde{a}(\zeta, \mu)=\langle\mu, g\rangle_{-1 / 2,1 / 2}$ for all $\mu \in Y$, where $g$ is given in $H^{1 / 2}(\tilde{\Gamma})$, has in principle no solution, except if $g$ is a trace of an harmonic function on $\tilde{\Gamma}$. It follows that if we define the operator 
$\tilde{R}: H^{1 / 2}(\Gamma) \rightarrow H^{1 / 2}(\tilde{\Gamma})$ by $\left(\tilde{R} u_{0}\right)(x)=\int_{\Gamma}\left(\partial G / \partial n_{y}\right)(x, y) u_{0}(y) \mathrm{d} s_{y}, \forall x \in \tilde{\Gamma}$, then the problem of finding $\zeta \in X$ such that

$$
\forall \mu \in Y: \tilde{a}(\zeta, \mu)=\left\langle\mu, \tilde{R} u_{0}\right\rangle_{-1 / 2,1 / 2}
$$

has a solution $\zeta$, but it is ill-posed. In the following, we attack the numerical approximation of problem (7) by using a Galerkin method. By splitting up $\Gamma$ and $\tilde{\Gamma}$ into $n+1$ simple arcs of curve $\Gamma_{1}, \Gamma_{2}, \ldots, \Gamma_{n+1}$ and $\tilde{\Gamma}_{1}, \tilde{\Gamma}_{2}, \ldots, \tilde{\Gamma}_{n+1}$, respectively, we can look for a piecewise constant function $\zeta$ on $\Gamma_{j}, 1 \leqslant j \leqslant n+1$, satisfying (7) for all piecewise constant function $\mu$ on $\tilde{\Gamma}_{j}, 1 \leqslant j \leqslant n+1$. Actually, this method does not work when $n$ is very large except if we choose $\tilde{\Gamma}$ very close to $\Gamma$. In practice, the Galerkin method is efficient when the distance between $\Gamma$ and $\tilde{\Gamma}$ is of the same order as the smallest arc of curve splitting up $\Gamma$ and $\tilde{\Gamma}$. For this reason it is necessary to choose $\tilde{\Gamma}$ by taking into account the degree of accuracy of the approximation. To do this we replace $\tilde{\Gamma}$ by $\left(\Gamma^{(n)}\right)_{n=1}^{\infty}$ which will be a family of curves $\Gamma^{(n)}$ surrounding $\Omega$ and converging to $\Gamma$ when $n$ tends to infinity. We split up $\Gamma$ and $\Gamma^{(n)}$ into $n+1$ simple arcs of curve denoted by $\Gamma_{1}, \Gamma_{2}, \ldots, \Gamma_{n+1}$ and $\Gamma_{1}^{(n)}, \Gamma_{2}^{(n)}, \ldots, \Gamma_{n+1}^{(n)}$, respectively, and we define

$$
\begin{aligned}
X_{n} & =\left\{\lambda \in H_{0}^{-1 / 2}(\Gamma):\left.\lambda\right|_{\Gamma_{i}} \text { is constant, } i=1,2, \ldots, n+1\right\} \\
Y_{n} & =\left\{\mu \in H_{0}^{-1 / 2}\left(\Gamma^{(n)}\right):\left.\lambda\right|_{\Gamma_{i}^{(n)}} \text { is constant, } i=1,2, \ldots, n+1\right\}
\end{aligned}
$$

The discrete problem corresponding to (7) consists in finding $\hat{\zeta}_{n} \in X_{n}$ such that

$$
\forall \mu_{n} \in Y_{n}: a_{n}\left(\hat{\zeta}_{n}, \mu_{n}\right)=\left\langle\mu_{n}, R_{n} u_{0}\right\rangle_{-1 / 2,1 / 2}
$$

where $a_{n}(.,$.$) is defined by \tilde{a}(.,$.$) in which \tilde{\Gamma}$ is replaced by $\Gamma^{(n)}$ and where $R_{n}: H^{1 / 2}(\Gamma) \rightarrow$ $H^{1 / 2}\left(\Gamma^{(n)}\right)$ is defined by

$$
\forall x \in \Gamma^{(n)}:\left(R_{n} u_{0}\right)(x)=\int_{\Gamma} \frac{\partial G}{\partial n_{y}}(x, y) u_{0}(y) \mathrm{d} s_{y}
$$

Remark that $\operatorname{dim} X_{n}=\operatorname{dim} Y_{n}=n$ and problem (8) is a linear system of $n$ equations for $n$ unknowns. A consequence of the fact that (7) is ill-posed is that often the approximate problem (8) is unstable. The goal of this paper is to establish how we have to choose $\Gamma^{(n)}$ and its splitting (together with the splitting of $\Gamma$ ) in order to obtain the stability of the numerical scheme (8) and the convergence of $\hat{\zeta}_{n}$ to $\partial u / \partial n_{y}$ when $n$ tends to infinity. We also establish error estimates between $\hat{\zeta}_{n}$ and $\partial u / \partial n_{y}$. Moreover, we give the rules for the choice of a quadrature formula when we want to numerically compute $\hat{\zeta}_{n}$. This quadrature rule does not affect the stability and convergence properties of the scheme. In particular, if $\zeta$ denotes the normal derivative of $u$ on $\Gamma$ then we have

$$
\left\|\hat{\zeta}_{n}-\zeta\right\|_{-1 / 2, \Gamma}=\mathcal{O}\left(\frac{1}{n^{3 / 2}}\right)
$$

Note that instead of (8) one can also consider the problem of finding $\zeta_{n} \in Y_{n}$ such that

$$
\forall \mu_{n} \in X_{n}: \int_{\Gamma} \mathrm{d} s_{x} \int_{\Gamma^{(n)}} \mathrm{d} s_{y} G(x, y) \zeta_{n}(y) \mu_{n}(x)=\int_{\Gamma} u_{0}(x) \mu_{n}(x) \mathrm{d} s_{x}
$$


In fact, problem (11) is a linear system in which appears the same matrix as in (8), and thus it is stable under the same conditions. Moreover, by using the arguments developed in this paper, one can prove that the same estimate (10) holds, where in this case $\zeta$ is the solution of the weakly singular single layer equation

$$
\forall \mu \in H_{0}^{-1 / 2}(\Gamma): \int_{\Gamma} \mathrm{d} s_{x} \int_{\Gamma} \mathrm{d} s_{y} G(x, y) \zeta(y) \mu(x)=\int_{\Gamma} u_{0}(x) \mu(x) \mathrm{d} s_{x}
$$

Thus, the proposed scheme can also be considered as a special quadrature formula method for the standard piecewise linear Galerkin approximation of the weakly singular single layer potential. Under this point of view, the method is related to a class that has been studied for many years, from the early papers [8,9] up to recent papers (Reference [10] for instance). Nevertheless, the special quadrature obtained here by introducing a neighbouring curve does not fall in a category which has been analysed in a previous work.

The numerical analysis we present here show that the efficiency of our scheme is asymptotically of the same order than the method currently used, but its major feature resides in the simplicity of the ideas used for its construction. It can be also used (but without rigorous justification) in the case when the curve has corners, and the same simple ideas can be applied in the 3D case (cf. Reference [11]). It may be the main reason which has motivated engineers to employ it. We point out that similar but unstable methods seem to have a certain success with engineers (see, for instance, References [12,13]).

In addition to the papers [8-10], for a review of classical boundary element methods, we refer to References [5,14-16]. In practice, the boundary element methods are often used in combination with finite element method (cf. Reference [17] for a reference article). We can see in References [18] or [19] how our method can be used with finite element methods in order to simulate a two-dimensional induction heating problem. Another interesting nonsingular method has been presented in Reference [20].

In the present paper, we analyse the only circular case where the Laplace problem is set on a disk. We obtain similar results when the Laplace problem is set outside the disk. In a second paper we use compact perturbation arguments to treat the general case.

\section{THE CIRCULAR CASE}

In this section, we assume that $\Gamma$ is a circle centred at the origin with radius $c$. The curves $\Gamma^{(n)}$ will also be some circles centred at the origin with radius $c_{n}>c$. We will use the complex notation to describe $\Gamma$ and $\Gamma^{(n)}$, i.e.

$$
\begin{aligned}
\Gamma & =\left\{z(t) \in \mathbb{C}: z(t)=c \mathrm{e}^{\mathrm{i} t}, t \in[0,2 \pi]\right\} \\
\Gamma^{(n)} & =\left\{z^{(n)}(t) \in \mathbb{C}: z^{(n)}(t)=c_{n} \mathrm{e}^{\mathrm{i} t}, t \in[0,2 \pi]\right\}
\end{aligned}
$$

The main factor which will appear in the following is the ratio $\gamma_{n}$ between $c_{n}$ and $c$, i.e. $\gamma_{n}=c_{n} / c$. In order to discretize $\Gamma$ and $\Gamma^{(n)}$, we set $h=2 \pi /(n+1), t_{j}=j h, j=0,1, \ldots, n+$ $1, t_{j+1 / 2}=(j+1 / 2) h, j=0,1, \ldots, n$. To these points we associate the corresponding points on 
$\Gamma$ and $\Gamma^{(n)}$, that is to say

$$
\begin{gathered}
z_{j}=c \mathrm{e}^{\mathrm{i} t_{j}}, \quad 0 \leqslant j \leqslant n+1, \quad z_{j+1 / 2}=c \mathrm{e}^{\mathrm{i} t_{j+1 / 2}}, 0 \leqslant j \leqslant n \\
z_{j}^{(n)}=c_{n} \mathrm{e}^{\mathrm{i} t_{j}}, \quad 0 \leqslant j \leqslant n+1, \quad z_{j+1 / 2}^{(n)}=c_{n} \mathrm{e}^{\mathrm{i} t_{j+1 / 2}}, \quad 0 \leqslant j \leqslant n
\end{gathered}
$$

Now we can define

$$
\begin{aligned}
\Gamma_{j} & =\left\{z(t) \in \mathbb{C}: z(t)=c \mathrm{e}^{\mathrm{i} t}, t \in\left[t_{j-1 / 2}, t_{j+1 / 2}\right]\right\}, \quad 1 \leqslant j \leqslant n \\
\Gamma_{n+1} & =\left\{z(t) \in \mathbb{C}: z(t)=c \mathrm{e}^{\mathrm{i} t}, t \in\left[t_{n+1 / 2}, 2 \pi\right] \cup\left[0, t_{1 / 2}\right]\right\} \\
\Gamma_{j}^{(n)} & =\left\{z^{(n)}(t) \in \mathbb{C}: z^{(n)}(t)=c_{n} \mathrm{e}^{\mathrm{i} t}, t \in\left[t_{n-1 / 2}, t_{n+1 / 2}\right]\right\}, \quad 1 \leqslant j \leqslant n \\
\Gamma_{n+1}^{(n)} & =\left\{z^{(n)}(t) \in \mathbb{C}: z^{(n)}(t)=c_{n} \mathrm{e}^{\mathrm{i} t}, t \in\left[t_{n+1 / 2}, 2 \pi\right] \cup\left[0, t_{1 / 2}\right]\right\}
\end{aligned}
$$

and we recall that

$$
\begin{aligned}
& X_{n}=\left\{\lambda \in H_{0}^{-1 / 2}(\Gamma):\left.\lambda\right|_{\Gamma_{i}} \text { is constant, } i=1,2, \ldots, n+1\right\} \\
& Y_{n}=\left\{\mu \in H_{0}^{-1 / 2}\left(\Gamma^{(n)}\right):\left.\mu\right|_{\Gamma_{i}^{(n)}} \text { is constant, } i=1,2, \ldots, n+1\right\}
\end{aligned}
$$

Our main result is the following:

Theorem 2.1. Assume that $\gamma_{n}=1+\delta / n$ where $\delta$ is a positive number. Then there exists $\delta_{0}>0$ such that for all $\left.\delta \in\right] 0, \delta_{0}$ [, problem (8) has a unique solution $\hat{\zeta}_{n} \in X_{n}$ for any integer $n$ and we have $\lim _{n \rightarrow \infty}\left\|\partial u / \partial n-\hat{\zeta}_{n}\right\|_{-1 / 2, \Gamma}=0$, where $u$ is the solution of (1).

Moreover, if $\partial u / \partial n \in H^{1}(\Gamma)$, there exists a constant $C$ independent of $n$ such that

$$
\left\|\frac{\partial u}{\partial n}-\hat{\zeta}_{n}\right\|_{-1 / 2, \Gamma} \leqslant \frac{C}{n^{3 / 2}}
$$

Before proving this theorem, we establish three technical lemmata. In the following we will use Fourier technical arguments and for this reason we will work in the complex spaces $H_{0}^{-1 / 2}(\Gamma), H_{0}^{-1 / 2}\left(\Gamma^{(n)}\right)$. We define the sesquilinear form $a: H_{0}^{-1 / 2}(\Gamma) \times H_{0}^{-1 / 2}(\Gamma) \rightarrow \mathbb{C}$ by

$$
\hat{\lambda}, \hat{\mu} \in H_{0}^{-1 / 2}(\Gamma): a(\hat{\lambda}, \hat{\mu})=\int_{\Gamma} \mathrm{d} s_{x} \int_{\Gamma} \mathrm{d} s_{y} G(x, y) \hat{\lambda}(y) \overline{\hat{\mu}(x)}
$$

where $\bar{\mu}$ is the complex conjugate of $\mu$. When $\mu \in H_{0}^{-1 / 2}\left(\Gamma^{(n)}\right)$ we set $\hat{\mu}(x)=\mu\left(\gamma_{n} x\right)$ for $x \in \Gamma$ and we have $\hat{\mu} \in H_{0}^{-1 / 2}(\Gamma)$. In the following $\tilde{a}(.,$.$) and a_{n}(.,$.$) are also considered as sesquilin-$ ear forms on the complexified Sobolev spaces $H^{-1 / 2}$.

Now we are in the position to set the eigenproblem which consists in looking for $\hat{\lambda} \in X_{n}$, $\hat{\lambda} \neq 0$ and $\omega \in \mathbb{C}$ satisfying

$$
\forall \mu \in Y_{n}: a_{n}(\hat{\lambda}, \mu)=\omega a(\hat{\lambda}, \hat{\mu})
$$


By setting $\hat{v}_{m}(z)=(z / c)^{m}, z \in \Gamma \subset \mathbb{C}$ and by defining $\hat{\Pi}_{n}: H^{1}(\Gamma) \rightarrow L^{2}(\Gamma)$ by

$$
\begin{aligned}
& \hat{\Pi}_{n} \hat{v}(z)=\hat{v}\left(z_{j}\right), \quad z \in \Gamma_{j}, \quad j=1,2, \ldots, n \\
& \hat{\Pi}_{n} \hat{v}(z)=\hat{v}\left(z_{n+1}\right), \quad z \in \Gamma_{n+1}
\end{aligned}
$$

we obtain the following main result:

Lemma 2.1. The eigenproblem (23) possesses the solutions

$$
\omega_{m}=\gamma_{n} \frac{\sum_{p \in \mathbb{Z}} \gamma_{n}^{-|m+p(n+1)|}|m+p(n+1)|^{-3}}{\sum_{p \in \mathbb{Z}}|m+p(n+1)|^{-3}}, \quad \hat{\lambda}_{m}=\hat{\Pi}_{n} \hat{v}_{m}, \quad m=1,2, \ldots, n
$$

Proof. We begin by setting $v_{k}(z)=\left(z / c_{n}\right)^{k}, z \in \Gamma^{(n)}$, and we calculate for any integer $m$ and $k \in \mathbb{Z}$ :

$$
\begin{aligned}
a_{n}\left(\hat{v}_{m}, v_{k}\right) & =\int_{\Gamma^{(n)}} \mathrm{d} s_{x} \int_{\Gamma} \mathrm{d} s_{y} G(x, y) \hat{v}_{m}(y) \overline{v_{k}(x)} \\
& =-\frac{c c_{n}}{2 \pi} \int_{0}^{2 \pi} \mathrm{d} s \int_{0}^{2 \pi} \log \left|c \mathrm{e}^{\mathrm{i} t}-c_{n} \mathrm{e}^{\mathrm{i} s}\right| \mathrm{e}^{\mathrm{i} m t} \mathrm{e}^{-\mathrm{i} k s} \mathrm{~d} t \\
& =-\frac{c c_{n}}{2 \pi} \int_{0}^{2 \pi} \mathrm{e}^{-\mathrm{i} k s} \mathrm{~d} s \int_{0}^{2 \pi} \log \left|c \mathrm{e}^{\mathrm{i}(t-s)}-c_{n}\right| \mathrm{e}^{\mathrm{i} m t} \mathrm{~d} t
\end{aligned}
$$

By using a change of variable we obtain

$$
\begin{aligned}
\int_{0}^{2 \pi} \log \left|c \mathrm{e}^{\mathrm{i}(t-s)}-c_{n}\right| \mathrm{e}^{\mathrm{i} m t} \mathrm{~d} t & =\int_{-s}^{2 \pi-s} \log \left|c \mathrm{e}^{\mathrm{i} \tau}-c_{n}\right| \mathrm{e}^{\mathrm{i} m(s+\tau)} \mathrm{d} \tau \\
& =\mathrm{e}^{\mathrm{i} m s} \int_{-\pi}^{\pi} \log \left|c \mathrm{e}^{\mathrm{i} \tau}-c_{n}\right| \mathrm{e}^{\mathrm{i} m \tau} \mathrm{d} \tau \\
& =\mathrm{e}^{\mathrm{i} m s} \int_{-\pi}^{\pi} \log \left|c^{2}+c_{n}^{2}-2 c c_{n} \cos (\tau)\right|^{1 / 2} \mathrm{e}^{\mathrm{i} m \tau} \mathrm{d} \tau
\end{aligned}
$$

By using an elementary result (see Reference [3]) we conclude that

$$
\int_{0}^{2 \pi} \log \left|c \mathrm{e}^{\mathrm{i}(t-s)}-c_{n}\right| \mathrm{e}^{\mathrm{i} m t} \mathrm{~d} t= \begin{cases}-\frac{\pi}{|m|} \gamma_{n}^{-|m|} \mathrm{e}^{\mathrm{i} m s} & \text { if } m \neq 0 \\ 2 \pi \log c_{n} & \text { if } m=0\end{cases}
$$

It follows that

$$
a_{n}\left(\hat{v}_{m}, v_{k}\right)=\frac{\pi}{|m|} \gamma_{n}^{-|m|} \delta_{k m} c c_{n} \quad \text { when } m \neq 0
$$


By an analogous calculation we obtain with $\hat{\mu}(x)=\mu\left(\gamma_{n} x\right), x \in \Gamma$ :

$$
a\left(\hat{v}_{m}, \hat{v}_{k}\right)=\frac{\pi}{|m|} \delta_{k m} c^{2} \quad \text { when } m \neq 0
$$

Let now $\chi_{j}$ be the characteristic function of $\left[t_{j-1 / 2}, t_{j+1 / 2}\right]$ if $0 \leqslant j \leqslant n$ and $\chi_{n+1}$ be the characteristic function of $\left[t_{0}, t_{1 / 2}\right] \cup\left[t_{n+1 / 2}, t_{n+1}\right]$. We verify that the complex Fourier series of $\chi_{j}$ is given by

$$
\begin{aligned}
\chi_{j}(t) & =\sum_{k=-\infty}^{\infty} c_{j k} \mathrm{e}^{\mathrm{i} k t} \\
c_{j k} & =\frac{\sin (k \pi /(n+1))}{k \pi} \mathrm{e}^{-\mathrm{i} k k_{j}}, \quad j=0,1, \ldots, n+1, k \neq 0 \\
c_{j 0} & =\frac{1}{n+1}, \quad j=0,1, \ldots, n+1
\end{aligned}
$$

Now we define $\Pi_{n} \mathrm{e}^{\mathrm{i} m t}$ by

$$
\Pi_{n} \mathrm{e}^{\mathrm{i} m t}=\sum_{j=1}^{n+1} \mathrm{e}^{\mathrm{i} m t_{j}} \chi_{j}(t)
$$

By replacing (27)-(29) into (30) we obtain from a standard calculation

$$
\Pi_{n} \mathrm{e}^{\mathrm{i} m t}=\frac{n+1}{\pi} \sin \left(\frac{m \pi}{n+1}\right) \sum_{p=-\infty}^{\infty} \frac{(-1)^{p}}{m+p(n+1)} \mathrm{e}^{\mathrm{i}(m+p(n+1)) t}, \quad 1 \leqslant m \leqslant n
$$

Now let $m$ be equal to $1,2, \ldots, n$. By using (31) and the definitions of $\hat{\Pi}_{n}$ and $\hat{v}_{m}$ we have

$$
\hat{\Pi}_{n} \hat{v}_{m}(z)=\frac{n+1}{\pi} \sin \left(\frac{m \pi}{n+1}\right) \sum_{p=-\infty}^{\infty} \frac{(-1)^{p}}{m+p(n+1)} \hat{v}_{m+p(n+1)}(z)
$$

for all $z \in \Gamma$.

It suffices to use (25) and (32) to show that if $1 \leqslant m, k \leqslant n$, we have

$$
a_{n}\left(\hat{\Pi}_{n} \hat{v}_{m}, \Pi_{n} v_{k}\right)=\left(\frac{n+1}{\pi}\right)^{2} \sin ^{2}\left(\frac{m \pi}{n+1}\right) \pi c c_{n} \sum_{p=-\infty}^{\infty} \frac{\gamma_{n}^{-|m+p(n+1)|}}{|m+p(n+1)|^{3}} \delta_{m k}
$$

By using (26) instead of (25), together with (32), we obtain

$$
a\left(\hat{\Pi}_{n} \hat{v}_{m}, \hat{\Pi}_{n} \hat{v}_{k}\right)=\left(\frac{n+1}{\pi}\right)^{2} \sin ^{2}\left(\frac{m \pi}{n+1}\right) \pi c^{2} \sum_{p=-\infty}^{\infty} \frac{1}{|m+p(n+1)|^{3}} \delta_{m k}
$$

By setting $\omega_{m}=\gamma_{n} \sum_{p \in \mathbb{Z}} \gamma_{n}^{-|m+p(n+1)|}|m+p(n+1)|^{-3} / \sum_{p \in \mathbb{Z}}|m+p(n+1)|^{-3}$ we obtain for all $1 \leqslant k, m \leqslant n$ :

$$
a_{n}\left(\hat{\Pi}_{n} \hat{v}_{m}, \Pi_{n} v_{k}\right)=\omega_{n} a\left(\hat{\Pi}_{n} \hat{v}_{m}, \hat{\Pi}_{n} \hat{v}_{k}\right)
$$


The fact that $\hat{\Pi}_{n} \hat{v}_{k}$ for $k=1,2, \ldots, n$ is a basis of $X_{n}$ which is orthogonal with respect to the scalar product $a(.,$.$) together with (35) proves our lemma.$

Remark 2.1. It is well known (see Reference [21] for instance) that $a(.,$.$) is a scalar$ product on $H_{0}^{-1 / 2}(\Gamma)$ and the norm $\|\hat{\lambda}\|=a(\hat{\lambda}, \hat{\lambda})^{1 / 2}$ is equivalent to $\|\hat{\lambda}\|_{-1 / 2, \Gamma}$. Moreover, from relations (25) and (26) we obtain that $a_{n}\left(\hat{v}_{m}, v_{k}\right)=\gamma_{n}^{1-|m|} a\left(\hat{v}_{m}, \hat{v}_{k}\right)$ for all $k$ and for all $m \neq 0$. Because $\gamma_{n}>1$ and $\left(\hat{v}_{m}\right)_{m \in \mathbb{Z}^{*}}$ is an Hilbertian basis of $H_{0}^{-1 / 2}(\Gamma)$, this last equality proves that $a_{n}(.,$.$) cannot satisfy a inf-sup condition on X \times Y$.

In order to have a family of curves $\left(\Gamma^{(n)}\right)_{n=1}^{\infty}$ which converges to $\Gamma$, we choose from now $c_{n}=\gamma_{n} c$ with

$$
\gamma_{n}=1+\frac{\delta}{n}
$$

where $\delta$ is a positive number. With this choice we have the following result:

Lemma 2.2. For all $\varepsilon>0$ there exists $\delta_{0}>0$ such that if $0<\delta<\delta_{0}$, then

$$
\sup _{\hat{\lambda} \in X_{n}} \frac{\left|a_{n}(\hat{\lambda}, \lambda)-a(\hat{\lambda}, \hat{\lambda})\right|}{a(\hat{\lambda}, \hat{\lambda})} \leqslant \varepsilon \quad \text { for all } n \geqslant 1
$$

where we denote by $\lambda$ the function belonging to $Y_{n}$ and defined by $\lambda(z)=\hat{\lambda}\left(\gamma_{n}^{-1} z\right), z \in \Gamma^{(n)}$.

Proof. In Lemma 2.1 we have seen that $\hat{\lambda}_{m}=\hat{\Pi}_{n} \hat{v}_{m}$ are the eigenvectors of (23) corresponding to the eigenvalues $\omega_{m}$ defined in (24). We set $\lambda_{m}()=.\hat{\lambda}_{m}\left(\gamma_{n}^{-1}\right.$.). When $\hat{\lambda} \in X_{n}$, we can write $\hat{\lambda}=\sum_{m=1}^{n} \alpha_{m} \hat{\lambda}_{m}$ because $\hat{\lambda}_{m}$ is an orthogonal basis of $X_{n}$ with respect to the scalar product $a(.,$.$) . Moreover, we have by using Lemma 2.1$ and relation (25):

$$
\begin{aligned}
\left|a_{n}(\hat{\lambda}, \lambda)-a(\hat{\lambda}, \hat{\lambda})\right| & =\left|\sum_{l, m=1}^{n} \alpha_{m} \alpha_{l}\left(a_{n}\left(\hat{\lambda}_{m}, \lambda_{l}\right)-a\left(\hat{\lambda}_{m}, \hat{\lambda}_{l}\right)\right)\right| \\
& =\left|\sum_{l, m=1}^{n}\left(1-\omega_{m}\right) \alpha_{m} \alpha_{l} a\left(\hat{\lambda}_{m}, \hat{\lambda}_{l}\right)\right|=\left|\sum_{m=1}^{n}\left(1-\omega_{m}\right) \alpha_{m}^{2} a\left(\hat{\lambda}_{m}, \hat{\lambda}_{m}\right)\right| \\
& \leqslant \max _{1 \leqslant m \leqslant n}\left|1-\omega_{m}\right| a(\hat{\lambda}, \hat{\lambda})
\end{aligned}
$$

In order to prove Lemma 2.2, it remains to show that for all $\varepsilon>0$ there exists $\delta_{0}>0$ such that if $0<\delta \leqslant \delta_{0}$, then we have

$$
\max _{1 \leqslant m \leqslant n}\left|1-\omega_{m}\right| \leqslant \varepsilon, \quad \forall n \geqslant 1
$$

where $\omega_{m}$ is explicitly given by (24).

Let $m$ be an integer in $[1, n]$ and define $\beta_{p}=|m+p(n+1)|$ with $p \in \mathbb{Z}$. Because we have the development $(1+x)^{-\beta_{p}}=1-x \beta_{p}(1+\tilde{x})^{-\left(1+\beta_{p}\right)}$, where $\tilde{x} \in(0, x), x>0$, we have the existence 
of a number $\eta_{p} \in(0, \delta)$ satisfying

$$
\left(1+\frac{\delta}{n}\right)^{-\beta_{p}}=1-\frac{\delta}{n} \beta_{p}\left(1+\frac{\eta_{p}}{n}\right)^{-\left(1+\beta_{p}\right)}
$$

Since $\gamma_{n}=1+\delta / n$, we have $1-\gamma_{n}^{-\beta_{p}}=\delta / n \beta_{p}\left(1+\eta_{p} / n\right)^{-\left(1+\beta_{p}\right)}$ and consequently

$$
\begin{aligned}
\gamma_{n}-\omega_{m} & =\gamma_{n}-\gamma_{n} \frac{\sum_{p=-\infty}^{\infty} \gamma_{n}^{-\beta_{p}} \beta_{p}^{-3}}{\sum_{p=-\infty}^{\infty} \beta_{p}^{-3}}=\gamma_{n}\left(1-\frac{\sum_{p=-\infty}^{\infty} \gamma_{n}^{-\beta_{p}} \beta_{p}^{-3}}{\sum_{p=-\infty}^{\infty} \beta_{p}^{-3}}\right) \\
& =\gamma_{n} \frac{\delta}{n} \frac{\sum_{p=-\infty}^{\infty}\left(1+\frac{\eta_{p}}{n}\right)^{-\left(1+\beta_{p}\right)} \beta_{p}^{-2}}{\sum_{p=-\infty}^{\infty} \beta_{p}^{-3}}
\end{aligned}
$$

It follows that

$$
\left|\gamma_{n}-\omega_{m}\right| \leqslant \gamma_{n} \frac{\delta}{n} \frac{\sum_{p=-\infty}^{\infty} \beta_{p}^{-2}}{\sum_{p=-\infty}^{\infty} \beta_{p}^{-3}}
$$

Now we evaluate an upper bound for $\sum_{p=-\infty}^{\infty} \beta_{p}^{-2}$ and a lower bound for $\sum_{p=-\infty}^{\infty} \beta_{p}^{-3}$.

- We have

$$
\begin{aligned}
\sum_{p=-\infty}^{\infty} \beta_{p}^{-2}= & \sum_{p \geqslant 0} \frac{1}{(m+p(n+1))^{2}}+\sum_{p \geqslant 1} \frac{1}{(p(n+1)-m)^{2}} \\
= & \frac{1}{(n+1)^{2}}\left(\sum_{p \geqslant 0} \frac{1}{(p+m /(n+1))^{2}}+\sum_{p \geqslant 1} \frac{1}{(p-m /(n+1))^{2}}\right) \\
= & \frac{1}{(n+1)^{2}}\left(\frac{(n+1)^{2}}{m^{2}}+\frac{(n+1)^{2}}{(n+1-m)^{2}}+\sum_{p \geqslant 1} \frac{1}{(p+m /(n+1))^{2}}\right. \\
& \left.+\sum_{p \geqslant 2} \frac{1}{(p-m /(n+1))^{2}}\right)
\end{aligned}
$$

By using the following inequalities:

$$
\sum_{p \geqslant 1} \frac{1}{(p+m /(n+1))^{2}} \leqslant \int_{m /(n+1)}^{\infty} \frac{\mathrm{d} x}{x^{2}} \text { and } \sum_{p \geqslant 2} \frac{1}{(p-m /(n+1))^{2}} \leqslant \int_{1-m /(n+1)}^{\infty} \frac{\mathrm{d} x}{x^{2}}
$$

we obtain

$$
\sum_{p=-\infty}^{\infty} \beta_{p}^{-2} \leqslant \frac{1}{m^{2}}+\frac{1}{(n+1-m)^{2}}+\frac{1}{n+1}\left(\frac{1}{m}+\frac{1}{n+1-m}\right)
$$


- In the same way, we have

$$
\sum_{p=-\infty}^{\infty} \beta_{p}^{-3}=\frac{1}{(n+1)^{3}}\left(\sum_{p \geqslant 0} \frac{1}{(p+m /(n+1))^{3}}+\sum_{p \geqslant 1} \frac{1}{(p-m /(n+1))^{3}}\right)
$$

and by using the following inequalities:

$$
\sum_{p \geqslant 0} \frac{1}{(p+m /(n+1))^{3}} \geqslant \int_{m /(n+1)}^{\infty} \frac{\mathrm{d} x}{x^{3}} \text { and } \sum_{p \geqslant 1} \frac{1}{(p-m /(n+1))^{3}} \geqslant \int_{1-m /(n+1)}^{\infty} \frac{\mathrm{d} x}{x^{3}}
$$

we obtain

$$
\sum_{p=-\infty}^{\infty} \beta_{p}^{-3} \geqslant \frac{1}{2(n+1)}\left(\frac{1}{m^{2}}+\frac{1}{(n+1-m)^{2}}\right)
$$

Due to (41) and (42) we obtain

$$
\frac{\sum_{p=-\infty}^{\infty} \beta_{p}^{-2}}{\sum_{p=-\infty}^{\infty} \beta_{p}^{-3}} \leqslant 2(n+1)\left(1+\frac{m(n+1-m)}{m^{2}+(n+1-m)^{2}}\right)
$$

By setting

$$
g(x)=\frac{x(n+1-x)}{x^{2}+(n+1-x)^{2}}=\frac{1}{2}\left(\frac{(n+1)^{2}}{x^{2}+(n+1-x)^{2}}-1\right)
$$

we easily show that $\max _{x \in[1, n]} g(x)=g((n+1) / 2) \leqslant \frac{1}{2}$. Consequently, relation (43) implies

$$
\frac{\sum_{p=-\infty}^{\infty} \beta_{p}^{-2}}{\sum_{p=-\infty}^{\infty} \beta_{p}^{-3}} \leqslant 3(n+1)
$$

By using (40), we finally obtain

$$
\left|\gamma_{n}-\omega_{m}\right| \leqslant 3 \gamma_{n} \delta \frac{n+1}{n} \leqslant 6 \delta \gamma_{n} \text { for all } n \geqslant 1
$$

It suffices to write $\gamma_{n}=1+\delta / n$ to conclude that (38) is true.

A corollary of Lemma 2.2 is

Lemma 2.3. By setting $\gamma_{n}=1+\delta / n$, there exists $\delta_{0}$ and $\alpha>0$ independent of $n$ such that for $0<\delta<\delta_{0}$ we have

$$
a_{n}(\hat{\lambda}, \lambda) \geqslant \alpha\|\hat{\lambda}\|_{-1 / 2, \Gamma}^{2}, \quad \forall \hat{\lambda} \in X_{n}, \forall n \geqslant 1
$$

Proof. This is a direct consequence of Lemma 2.2 together with Remark 2.1.

Proof of Theorem 2.1. Lemma 2.3 implies an uniform 'inf-sup' condition for $\delta \in] 0, \delta_{0}[$, i.e.

$$
\inf _{\substack{\hat{\lambda} \in X_{n} \\\|\hat{\lambda}\|_{-1 / 2, \Gamma}=1}} \sup _{\substack{\mu \in Y_{n} \\\|\mu\|_{-1 / 2, \Gamma(n)=1}}} a_{n}(\hat{\lambda}, \mu) \geqslant \alpha, \quad n \geqslant 1
$$


where $\alpha$ is a positive constant independent of $n$. It follows (see Reference [7] for instance) that problem (8) has a unique solution $\hat{\zeta}_{n} \in X_{n}$. By setting $\hat{\zeta}=\partial u / \partial n$ on $\Gamma$, where $u$ is solution of (1), we have seen that for all $\mu \in H_{0}^{-1 / 2}\left(\Gamma^{(n)}\right)$ we obtain $a_{n}(\hat{\zeta}, \mu)=\left\langle\mu, R_{n} u_{0}\right\rangle$, and consequently for all $\mu_{n} \in Y_{n}$ we have

$$
a_{n}\left(\hat{\zeta}-\hat{\zeta}_{n}, \mu_{n}\right)=0
$$

Let now $\hat{\varphi}_{n}$ be any function of $X_{n}$. By using Lemma 2.3 and (48), we have

$$
\left\|\hat{\zeta}_{n}-\hat{\varphi}_{n}\right\|_{-1 / 2, \Gamma} \leqslant \frac{1}{\alpha} a_{n}\left(\hat{\zeta}_{n}-\hat{\varphi}_{n}, \zeta_{n}-\varphi_{n}\right)=\frac{1}{\alpha} a_{n}\left(\hat{\zeta}-\hat{\varphi}_{n}, \zeta_{n}-\varphi_{n}\right)
$$

where we denote by $\zeta_{n}(z)=\hat{\zeta}\left(\gamma_{n}^{-1} z\right)$ and $\varphi_{n}(z)=\hat{\varphi}_{n}\left(\gamma_{n}^{-1} z\right), \forall z \in \Gamma^{(n)}$.

From inequality (49) and from the uniform continuity of $a_{n}$ with respect to $n$ (see Reference [18, pp. 34-35]), we deduce there exists a constant $C$, independent of $n$, such that

$$
\left\|\hat{\zeta}_{n}-\hat{\varphi}_{n}\right\|_{-1 / 2, \Gamma} \leqslant C\left\|\hat{\zeta}-\hat{\varphi}_{n}\right\|_{-1 / 2, \Gamma}
$$

By using the triangular inequality we finally obtain

$$
\left\|\hat{\zeta}-\hat{\zeta}_{n}\right\|_{-1 / 2, \Gamma} \leqslant(1+C)\left\|\hat{\zeta}-\hat{\varphi}_{n}\right\|_{-1 / 2, \Gamma}
$$

and consequently

$$
\left\|\hat{\zeta}-\hat{\zeta}_{n}\right\|_{-1 / 2, \Gamma} \leqslant(1+C) \min _{\hat{\varphi}_{n} \in X_{n}}\left\|\hat{\zeta}-\hat{\varphi}_{n}\right\|_{-1 / 2, \Gamma}
$$

The proof of Theorem 2.1 is a consequence of the fact that $\overline{\bigcup_{n=1}^{\infty} X_{n}}=H_{0}^{-1 / 2}(\Gamma)$ and from the following estimate (see Reference [15] for instance):

$$
\left\|\hat{\zeta}-P_{n} \hat{\zeta}\right\|_{-1 / 2, \Gamma} \leqslant \frac{C}{n^{3 / 2}}\|\hat{\zeta}\|_{1, \Gamma}
$$

when $P_{n}$ is the orthogonal projector of $L^{2}(\Gamma)$ onto $X_{n}$.

\section{ERRORS ESTIMATES WITH NUMERICAL INTEGRATION}

In order to solve the discrete problem (8) in the circular case situation, we will have to build the matrix of coefficients $A_{i j}=a_{n}\left(\hat{\varphi}_{i}, \varphi_{j}\right), 1 \leqslant i, j \leqslant n$, where $\left(\hat{\varphi}_{i}\right)_{i=1}^{n}$ is a finite element basis of $X_{n}$. It follows that we will have to calculate expressions of the form

$$
\int_{\Gamma_{k}^{(n)}} \mathrm{d} s_{x} \int_{\Gamma_{l}} \mathrm{~d} s_{y} G(x, y)=-\frac{c c_{n}}{2 \pi} \int_{t_{k-1 / 2}}^{t_{k+1 / 2}} \mathrm{~d} s \int_{t_{l-1 / 2}}^{t_{l+1 / 2}} \mathrm{~d} t \log \left|c \mathrm{e}^{\mathrm{i} t}-c_{n} \mathrm{e}^{\mathrm{i} s}\right|
$$

since $\left(\hat{\varphi}_{i}\right)_{i=1}^{n}$ are constant on $\Gamma_{k}$. Clearly speaking we take $c_{n}=\gamma_{n} c$ with $\gamma_{n}=1+\delta / n$ and for $n$ sufficiently large, it is difficult to numerically evaluate the quantity $\int_{t_{k-1 / 2}}^{t_{k+1 / 2}} \mathrm{~d} s \int_{t_{l-1 / 2}}^{t_{l+1 / 2}} \mathrm{~d} t \log \mid c \mathrm{e}^{\mathrm{i} t}-$ $c_{n} \mathrm{e}^{\mathrm{i} s} \mid$ when $k=l, l+1$ or $l-1$, because the integrand is almost singular. Remark that we have the same problem when we want to compute the right-hand side of (8). In the following, we give a rule in order to numerically perform this type of integrals by keeping the rate 
of accuracy $\mathcal{O}\left(1 / n^{3 / 2}\right)$ in $H_{0}^{-1 / 2}(\Gamma)$. Recall that the length of the interval $\left[t_{k-1 / 2}, t_{k+1 / 2}\right]$ is $h$, with $h=2 \pi /(n+1)$, and consider, for a positive integer $m$, the points in $[0,2 \pi]$ defined by $t_{k, j}=t_{k-1 / 2}+j(h / m) \bmod 2 \pi, 0 \leqslant j \leqslant m, 1 \leqslant k \leqslant n+1$.

For a continuous function $f(s, t)$ on $[0,2 \pi] \times[0,2 \pi]$ (for instance $f(s, t)=\log \left|c \mathrm{e}^{\mathrm{i} t}-c_{n} \mathrm{e}^{\mathrm{i} s}\right|$ ) we have

$$
\int_{t_{k-1 / 2}}^{t_{k+1 / 2}} \mathrm{~d} s \int_{t_{l-1 / 2}}^{t_{l+1 / 2}} \mathrm{~d} t f(s, t)=\sum_{i=0}^{m-1} \sum_{j=0}^{m-1} \int_{t_{k i}}^{t_{k, i+1}} \mathrm{~d} s \int_{t_{l j}}^{t_{l, j+1}} \mathrm{~d} t f(s, t)
$$

and by changing the variables $s$ and $t$ :

$$
\int_{t_{k-1 / 2}}^{t_{k+1 / 2}} \mathrm{~d} s \int_{t_{l-1 / 2}}^{t_{l+1 / 2}} f(s, t)=\frac{h^{2}}{4 m^{2}} \sum_{i=0}^{m-1} \sum_{j=0}^{m-1} \int_{-1}^{1} \mathrm{~d} \xi \int_{-1}^{1} \mathrm{~d} \eta f^{k_{i}, l_{j}}(\xi, \eta)
$$

with $f^{k_{i}, l_{j}}(\xi, \eta)=f\left(t_{k, i}+(\xi+1) / 2(h / m), t_{l, j}+(\eta+1) / 2(h / m)\right)$.

Let now $J(g)=\sum_{p=1}^{r} w_{p} g\left(\xi_{p}\right)$ be a numerical quadrature formula with $r$ integration points $-1 \leqslant \xi_{1}<\xi_{2}<\cdots<\xi_{r} \leqslant 1$ and weights $w_{1}, w_{2}, \ldots, w_{r}$. We assume that this formula is exact for polynomials $\mathbb{P}_{M}$ of degree $M$, that is to say for all $g \in \mathbb{P}_{M}$ we have

$$
J(g)=\int_{-1}^{1} g(\xi) \mathrm{d} \xi
$$

An approximation of (53) will be

$$
\int_{t_{k-1 / 2}}^{t_{k+1 / 2}} \mathrm{~d} s \int_{t_{l-1 / 2}}^{t_{l+1 / 2}} \mathrm{~d} t f(s, t) \approx \frac{h^{2}}{4 m^{2}} \sum_{i=0}^{m-1} \sum_{j=0}^{m-1} \sum_{p=1}^{r} \sum_{q=1}^{r} w_{p} w_{q} f^{k_{i}, l_{j}}\left(\xi_{p}, \xi_{q}\right)
$$

We set $I_{n k l}(f)$ as the left-hand side of (55) and $J_{n k l}(f)$ as its right-hand side. Then it is well known that if $f \in \mathscr{C}^{M+1}\left(\bar{\Omega}_{k l}\right)$ with $\Omega_{k l}=\left(t_{k-1 / 2}, t_{k+1 / 2}\right) \times\left(t_{l-1 / 2}, t_{l+1 / 2}\right)$, then we have the following estimate:

$$
\left|I_{n k l}(f)-J_{n k l}(f)\right| \leqslant C h^{2}\left(\frac{h}{m}\right)^{M+1} \max _{\alpha_{1}+\alpha_{2}=M+1} \max _{(s, t) \in \Omega_{k l}}\left|\frac{\partial^{M+1} f}{\partial s^{\alpha_{1}} \partial t^{\alpha_{2}}}(s, t)\right|
$$

where $C$ is a constant independent of $n, m$ and $f$ but is depending on $M$.

Now we are able to establish an approximation of the discrete problem (8) by using above quadrature formulas. To this end, we define in a similar way as (2)-(7), for $\hat{\zeta} \in X_{n}, \mu \in Y_{n}$ :

$$
\begin{aligned}
N(\hat{\zeta}, \mu)(s, t) & =-\frac{c c_{n}}{2 \pi} \log \left|c \mathrm{e}^{\mathrm{i} t}-c_{n} \mathrm{e}^{\mathrm{i} s}\right| \hat{\zeta}\left(c \mathrm{e}^{\mathrm{i} t}\right) \mu\left(c_{n} \mathrm{e}^{\mathrm{i} s}\right) \\
\tilde{a}_{n}(\hat{\zeta}, \mu) & =\sum_{k, l=1}^{n+1} J_{n k l}(N(\hat{\zeta}, \mu)) \\
S(\mu)(s, t) & =-\frac{c c_{n}}{2 \pi} \frac{\left(c_{n} \mathrm{e}^{\mathrm{i} s}-c \mathrm{e}^{\mathrm{i} t}, \mathrm{e}^{\mathrm{i} t)}\right.}{\left|c_{n} \mathrm{e}^{\mathrm{i} s}-c \mathrm{e}^{\mathrm{i} t}\right|^{2}} u_{0}\left(c \mathrm{e}^{\mathrm{i} t}\right) \mu\left(c_{n} \mathrm{e}^{\mathrm{i} s}\right)
\end{aligned}
$$




$$
\tilde{b}_{n}(\mu)=\sum_{k, l=1}^{n+1} J_{n k l}(S(\mu))
$$

where we denote by $(.,$.$) the scalar product in \mathbb{R}^{2}=\mathbb{C}$, i.e.

$$
\left(c_{n} \mathrm{e}^{\mathrm{i} s}-c \mathrm{e}^{\mathrm{i} t}, \mathrm{e}^{\mathrm{i} t}\right)=c_{n}(\cos t \cos s+\sin t \sin s)-c=c_{n} \cos (t-s)-c
$$

With these notations, the approximate problem with quadrature formula consists in finding $\tilde{\zeta}_{n} \in X_{n}$ satisfying

$$
\tilde{a}_{n}\left(\tilde{\zeta}_{n}, \mu_{n}\right)=\tilde{b}_{n}\left(\mu_{n}\right), \quad \forall \mu_{n} \in Y_{n}
$$

Now we are able to establish our main result.

Theorem 3.1. Assume that $\gamma_{n}=1+\delta / n$ where $\delta$ is a positive number and $u_{0} \in \mathscr{C}^{M+1}(\Gamma)$. Moreover assume that $m=n^{\beta}$ with $\beta \geqslant 3 /(M+1)$. Then, there exists $\delta_{0}>0$ and $N_{0}>0$ such that for all $\delta \in] 0, \delta_{0}\left[\right.$, problem (62) has a unique solution $\tilde{\zeta}_{n} \in X_{n}$ for any integer $n \geqslant N_{0}$. Moreover, if $\partial u / \partial n \in H^{1}(\Gamma)$, then there exists a constant $C$ independent of $n$ such that

$$
\left\|\frac{\partial u}{\partial n}-\tilde{\zeta}_{n}\right\|_{-1 / 2, \Gamma} \leqslant \frac{C}{n^{3 / 2}}
$$

Before proving this result, we first establish:

Lemma 3.1. We assume that $c_{n}=c(1+\delta / n)$ with $\delta>0$ and that the quadrature formula is exact for polynomials of degree $M$. Then, there exists a constant $C$ independent of $n$ and $m$ such that for all $\hat{\lambda} \in X_{n}, \mu \in Y_{n}$ we have

$$
\begin{gathered}
\left|a_{n}(\hat{\lambda}, \mu)-\tilde{a}_{n}(\hat{\lambda}, \mu)\right| \leqslant C m^{-(M+1)}\|\hat{\lambda}\|_{L^{2}(\Gamma)}\|\mu\|_{L^{2}\left(\Gamma^{(n)}\right)} \\
\left|b_{n}(\mu)-\tilde{b}_{n}(\mu)\right| \leqslant C n m^{-(M+1)}\|\mu\|_{L^{2}\left(\Gamma^{(n)}\right)}
\end{gathered}
$$

where $b_{n}(\mu)=\int_{\Gamma^{(n)}} \mathrm{d} s_{x} \int_{\Gamma} \mathrm{d} s_{y}\left(\partial G / \partial n_{y}\right)(x, y) u_{0}(y) \mu(x)$.

Proof. In order to prove (62), we begin by remarking that

$$
a_{n}(\hat{\lambda}, \mu)=\sum_{k, l=1}^{n+1} I_{n k l}(N(\hat{\lambda}, \mu))
$$

By substraction with (58) we obtain

$$
\left|a_{n}(\hat{\lambda}, \mu)-\tilde{a}_{n}(\hat{\lambda}, \mu)\right| \leqslant \sum_{k, l=1}^{n+1}\left|I_{n k l}(N(\hat{\lambda}, \mu))-J_{n k l}(N(\hat{\lambda}, \mu))\right|
$$

Now we use the error estimate (56) and we have

$$
\left|a_{n}(\hat{\lambda}, \mu)-\tilde{a}_{n}(\hat{\lambda}, \mu)\right| \leqslant C h^{2}\left(\frac{h}{m}\right)^{M+1} \sum_{k, l=1}^{n+1} \max _{\alpha_{1}+\alpha_{2}=M+1} \max _{(s, t) \in \Omega_{k l}}\left|\frac{\partial^{M+1} N(\hat{\lambda}, \mu)}{\partial s^{\alpha_{1}} \partial t^{\alpha_{2}}}(s, t)\right|
$$


Since $\hat{\lambda}\left(c \mathrm{e}^{\mathrm{i} t}\right) \mu\left(c_{n} \mathrm{e}^{\mathrm{i} s}\right)$ is constant on $\Omega_{k l}$, we verify, by derivating $(M+1)$ times the function $\log \left|c \mathrm{e}^{\mathrm{i} t}-c_{n} \mathrm{e}^{\mathrm{i} t}\right|$, that there exists $C$ such that

$$
\max _{\alpha_{1}+\alpha_{2}=M+1} \max _{(s, t) \in \Omega_{k l}}\left|\frac{\partial^{M+1} N(\hat{\lambda}, \mu)}{\partial s^{\alpha_{1}} \partial t^{\alpha_{2}}}(s, t)\right| \leqslant C n^{M+1}\left|\hat{\lambda}\left(c \mathrm{e}^{\mathrm{i} t_{l}}\right)\right|\left|\mu\left(c_{n} \mathrm{e}^{\mathrm{i} t_{k}}\right)\right|
$$

By using a Cauchy-Schwartz inequality together with the fact that the measure of $\Gamma_{l}^{(n)}$ and $\Gamma_{k}$ is of order $1 / n$, we finally obtain

$$
\begin{aligned}
\left|a_{n}(\hat{\lambda}, \mu)-\tilde{a}_{n}(\hat{\lambda}, \mu)\right| & \leqslant C h^{2}\left(\frac{h}{m}\right)^{M+1} n^{M+2}\left(\sum_{l=1}^{n+1} \mid \hat{\lambda}\left(\left.c \mathrm{e}^{\mathrm{i} t_{l}}\right|^{2}\right)^{1 / 2}\left(\sum_{k=1}^{n+1} \mid \mu\left(\left.c_{n} \mathrm{e}^{\mathrm{i} t_{k}}\right|^{2}\right)^{1 / 2}\right.\right. \\
& \leqslant C h^{2}\left(\frac{h}{m}\right)^{M+1} n^{M+3}\|\hat{\lambda}\|_{L^{2}(\Gamma)} \cdot\|\mu\|_{L^{2}\left(\Gamma^{(n)}\right)}
\end{aligned}
$$

It suffices to see that $h=2 \pi /(n+1)$ in order to prove $(63)$.

In order to prove (64), we remark that for $\mu \in Y_{n}$ :

$$
b_{n}(\mu)-\tilde{b}_{n}(\mu)=\sum_{k, l=1}^{n+1}\left(I_{n k l}(S(\mu))-J_{n k l}(S(\mu))\right)
$$

and we use the same arguments as above after showing that $\max _{(s, t) \in \Omega_{k l}}\left|\left(\partial^{M+1} / \partial s^{\alpha_{1}} \partial s^{\alpha_{2}}\right) S(\mu)\right|$ is bounded by $C n^{M+2}\left|\mu\left(c_{n} \mathrm{e}^{\mathrm{i} t_{k}}\right)\right|$.

Proof of Theorem 3.1. By using Lemma 2.3 we can conclude there exists $\delta_{0}$ and $\alpha>0$ such that for $0<\delta<\delta_{0}$ we have

$$
a_{n}(\hat{\lambda}, \lambda) \geqslant \alpha\|\hat{\lambda}\|_{-1 / 2, \Gamma}^{2}, \quad \forall \hat{\lambda} \in X_{n}, \quad \forall n \geqslant 1
$$

By using Lemma 3.1 with $m=n^{\beta}$ and $\beta \geqslant 3 /(M+1)$ we obtain

$$
\left|a_{n}(\hat{\lambda}, \lambda)-\tilde{a}_{n}(\hat{\lambda}, \lambda)\right| \leqslant \frac{C}{n^{3}}\|\hat{\lambda}\|_{L^{2}(\Gamma)}^{2}, \quad \forall \hat{\lambda} \in X_{n}, \quad \forall n \geqslant 1
$$

By considering the inverse inequality (see Reference [15] for instance)

$$
\|\hat{\lambda}\|_{L^{2}(\Gamma)} \leqslant C \sqrt{n}\|\hat{\lambda}\|_{-1 / 2, \Gamma}
$$

we have

$$
\left|a_{n}(\hat{\lambda}, \lambda)-\tilde{a}_{n}(\hat{\lambda}, \lambda)\right| \leqslant \frac{C}{n^{2}}\|\hat{\lambda}\|_{-1 / 2, \Gamma}^{2}, \quad \forall \hat{\lambda} \in X_{n}, \forall n \geqslant 1
$$

By using (69) and (71), we obtain the existence of $N_{0}$ such that the following relation is satisfied:

$$
\tilde{a}_{n}(\hat{\lambda}, \lambda) \geqslant \frac{\alpha}{2}\|\hat{\lambda}\|_{-1 / 2, \Gamma}^{2}, \quad \forall \hat{\lambda} \in X_{n}, \forall n \geqslant N_{0}
$$

It follows that for $n \geqslant N_{0}$, the sesquilinear form $\tilde{a}_{n}(.,$.$) satisfies an 'inf-sup' condition on$ $X_{n} \times Y_{n}$ and, consequently, the existence and uniqueness of solution of (62) is proven. 
By a similar calculation we obtain from (64) and (70):

$$
\left|\tilde{b}_{n}(\mu)-b_{n}(\mu)\right| \leqslant \frac{C}{n^{3 / 2}}\|\mu\|_{-1 / 2, \Gamma^{(n)}}, \quad \forall \mu \in Y_{n}, \forall n \geqslant 1
$$

Now we are able to prove the error estimate of Theorem 3.1. Actually, we have

$$
a_{n}\left(\hat{\zeta}_{n}, \mu_{n}\right)=b_{n}\left(\mu_{n}\right), \quad \forall \mu_{n} \in Y_{n}
$$

and

$$
\tilde{a}_{n}\left(\tilde{\zeta}_{n}, \mu_{n}\right)=\tilde{b}_{n}\left(\mu_{n}\right), \quad \forall \mu_{n} \in Y_{n}
$$

It follows that for all $\mu_{n} \in Y_{n}$ :

$$
\tilde{a}_{n}\left(\hat{\zeta}_{n}-\tilde{\zeta}_{n}, \mu_{n}\right)=-\tilde{b}_{n}\left(\mu_{n}\right)+b_{n}\left(\mu_{n}\right)-a_{n}\left(\hat{\zeta}_{n}, \mu_{n}\right)+\tilde{a}_{n}\left(\hat{\zeta}_{n}, \mu_{n}\right)
$$

By using (72) together with (63), (70), (73) and (74) in which we take $\mu_{n}=\zeta_{n}-\tilde{\zeta}_{n}^{\prime},\left(\tilde{\zeta}_{n}^{\prime}()=.\tilde{\zeta}_{n}\right.$ $\left.\left(\gamma_{n}^{-1}.\right)\right)$, we obtain the existence of a constant $C$ such that for $n \geqslant N_{0}$ :

$$
\left\|\hat{\zeta}_{n}-\tilde{\zeta}_{n}\right\|_{-1 / 2, \Gamma} \leqslant C\left(\frac{1}{n^{3 / 2}}+\frac{1}{n^{2}}\left\|\hat{\zeta}_{n}\right\|_{-1 / 2, \Gamma}\right)
$$

It remains to use Theorem 2.1 in order to obtain the error estimate of Theorem 3.1.

Remark 3.1. By choosing $\beta=1$, that is to say we divide each interval $\left[t_{k-1 / 2}, t_{k+1 / 2}\right]$ by $n$ parts, it suffices to choose a quadrature formula exact for polynomials of degree $M=2$ in order to keep the rate of accuracy $1 / n^{3 / 2}$ in the norm $H_{0}^{-1 / 2}(\Gamma)$. It follows that Simpson quadrature formula is sufficient.

Remark 3.2. If we restrict the quadrature to be a Gauss-Legendre formula (with $r$ points), then we have $M+1=2 r$. In this case, the numerical evaluations of all integrals require $\left(n^{\beta+1} . r\right)^{2}$ computations. Since Theorem 3.1 says that it suffices to take $\beta \geqslant 3 / 2 r$ to keep the rate of accuracy $\mathcal{O}\left(1 / n^{3 / 2}\right)$, we have interest to take $\beta$ small enough. By choosing $\beta=0.1$ and $r=3 / 2 \beta=15$ we obtain $m \approx 2$ if $n=10^{3}$ and $m \approx 3$ if $n=10^{5}$. This result shows that in practice, it suffices to split each intervals $\left[t_{k-1 / 2}, t_{k+1 / 2}\right]$ into three parts and to use a GaussLegendre formula with 15 points.

Remark 3.3. In practice, it suffices to split only the 'critical' intervals $\left[t_{k-1 / 2}, t_{k+1 / 2}\right]$ and $\left[t_{l-1 / 2}, t_{l+1 / 2}\right]$ for $l=k, k-1, k+1$ when we numerically compute (52).

\section{NUMERICAL RESULTS}

In the circular case, the practical interest of our method is limited, but we conclude this article by a brief example.

Let $\Omega$ be the disk of radius two centred at the origin, and $u$ the function defined on $\Omega$ by $u\left(x_{1}, x_{2}\right)=\mathrm{e}^{x_{1}} \cos \left(x_{2}\right),\left(x_{1}, x_{2}\right) \in \Omega$. Then $u$ is harmonic on $\Omega$ and $\partial u /\left.\partial n\right|_{\Gamma}$ is the exact solution of (2) when we put $u_{0}=\left.u\right|_{\Gamma}$. On the other hand, setting $u_{0}=\left.u\right|_{\Gamma}$, we can calculate, for any integer $n$, the function $\tilde{\zeta}_{n}$ which is the solution of (62) when we split each interval into three 


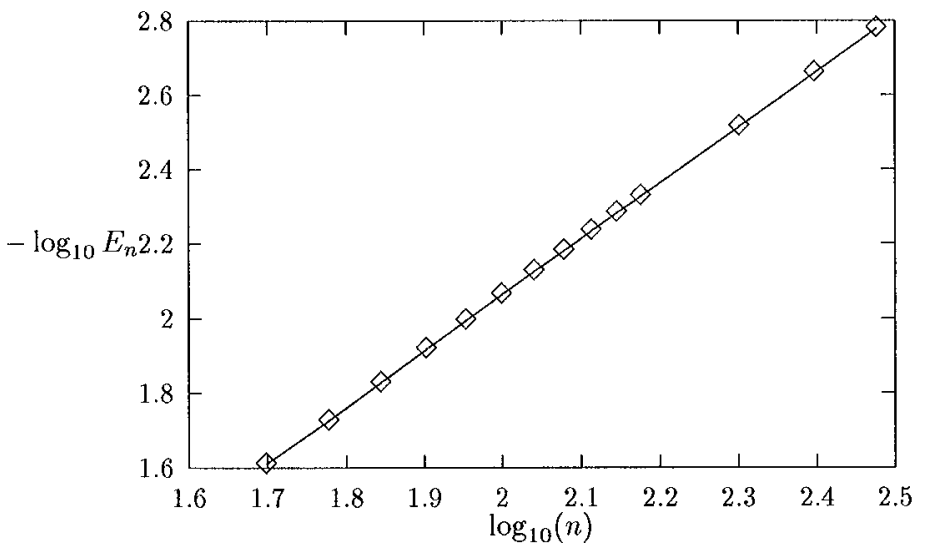

Figure 1. Relative $L^{2}$ error.

parts and we use a Gauss-Legendre formula with 15 points. For an integer $n$, we denote by $E_{n}=\left\|\partial u / \partial n-\tilde{\zeta}_{n}\right\|_{L^{2}} /\|\partial u / \partial n\|_{L^{2}}$, the relative $L^{2}$ error between the known exact solution and its approximation by our method.

In Figure 1, we have represented $-\log _{10} E_{n}$ in function of $\log _{10} n$. We can see that the curve obtained is a straight line with a slope approximatively equal to 1.5 . By using the inverse inequalities (cf. 70) in $X_{n}$, we can see that this experimental result is a little better than expected (slope equal one) by Theorem 3.1 .

\section{ACKNOWLEDGEMENTS}

Dreyfuss was supported by the Swiss National Fund for Scientific Research.

\section{REFERENCES}

1. Sloan IH. The Galerkin method for integral equation of the first kind with logarithmic kernel: Theory. IMA Journal of Numerical Analysis 1988; 8:105-122.

2. Kupradze VD. Dynamical problems in elasticity. In Progress in Solid Mechanics, Sneddon JN, Hill R (eds), Amsterdam: North Holland, vol. 3. 1963; 1-259.

3. Christiansen S. On Kupradze's functionnal equations for plane harmonic problems. In Function Theoric Methods in Differential Equations, Research Notes in Mathematics, Gilbert RP, Weinacht RJ (eds), Pitman Publishing: London, San Francisco, Del Barne, vol. 8. 1976; 205-243.

4. Christiansen S. Condition number of matrices derived from two classes of integral equations. Mathematical Methods in the Applied Sciences 1981; 3:364-392.

5. Kress R. Linear Integral Equations. Springer: Berlin, 1999.

6. Wearing JL, Sheikh MA. A regular indirect boundary element method for thermal analysis. International Journal of Numerical Methods in Engineering 1988; 25:495-515.

7. Babuska I, Aziz AK. The Mathematical Foundations of the Finite Element Method with Applications to Partial Differential Equations. Academic Press: New York, 1972.

8. Hsiao GC, Kopp P, Wendland WL. A Galerkin-collocation method for boundary integral equations of the first kind. Computing 1980; 25:89-130.

9. Hsiao GC, Kopp P, Wendland WL. Some applications of a Galerkin-collocation method for boundary integral equations of the first kind. Mathematical Methods in the Applied Sciences 1984; 6:280-325.

10. McLean W, Sloan IH. A fully discrete and symmetric boundary element method. IMA Journal of Numerical Analysis 1994; 14:311-345. 
11. Descloux J, Flueck M, Romério M. Modelling for instabilities in Hall-Héroult cells: mathematical and numerical aspects. Private Communication, Ecole Polytechnique Fédérale de Lausanne, 2000.

12. Golberg MA, Chen CS. The method of fundamental solutions for potential, Helmholtz and diffusion problems. In Boundary Integral Methods, Numerical and Mathematical Aspects. Computational Mechanics publications: Witt Press: Boston, Southampton, 1998.

13. Katsurada M. Asymptotic error analysis of the charge simulation method in a Jordan region with analytic boundary. Journal of the Faculty of Science, University of Tokyo Section IA. Mathematics 1990; 37: 635-657.

14. Sloan IH. Error analysis of boundary integral methods. Acta Numerica 1992; 1:287-339.

15. Prossdorf S, Silbermann B. Numerical Analysis for Integral and Related Equations. Birkhauser: Basel, 1991.

16. Nédélec JC. Approximation des équations intégrales em mécanique et en physique. Centre de mathématiques appliquées, Ecole Polytechnique de Palaiseau, 1977.

17. Johnson C, Nédélec JC. On the coupling of boundary integral and finite element methods. Mathematics of Computation 1980; 35(152):1063-1079.

18. Dreyfuss P. Analyse numérique d'une méthode intégrale frontière sans singularité-Application à l'électromagnétisme, Thèse no. 2049, Ecole Polytechnique Fédérale de Lausanne, 1999.

19. Dreyfuss P, Rappaz J. Numerical modelling of induction heating for two dimensional geometries, Preprint EPFL, 2001, to be submitted.

20. Lenoir M, Vullierme-Ledard M, Hazard C. Variational formulations for the determination of resonant states in scattering problems. SIAM Journal on Mathematical Analysis 1992; 23(3):579-608.

21. Le Roux MN. Méthode d'éléments finis pour la résolution numérique de problèmes extérieurs en dimension 2. RAIRO, Analyse Numérique 1977; 11(1):27-66. 